\title{
Contribution of neuroinflammation to the development of Alzheimer's disease-like pathology in OXYS rats
}

\author{
O.Yu. Koneva ${ }^{1,2 *}$, E.A. Rudnitskaya ${ }^{1}$, Yu.V. Rumyantseva ${ }^{1}$, N.A. Stefanova ${ }^{1}$ \\ ${ }^{1}$ Institute of Cytology and Genetics SB RAS, Novosibirsk, Russia \\ ${ }^{2}$ Novosibirsk State University, Novosibirsk, Russia \\ *e-mail: okoneva94@gmail.com
}

Key words: Alzheimer's disease, neuroinflammation, RNA-Seq, OXYS rats

Motivation and Aim: Brain aging is central to Alzheimer's disease (AD), although the mechanisms by which it occurs are not fully understood. In the last decade, accumulating evidence in support of a role of age-related dysregulation of neuroinflammation and immune system in AD. It remains unclear whether astrocytes and immune cells primarily microglia - influence disease onset, progression or both. Using senescenceaccelerated OXYS rats, which simulate key characteristics of sporadic AD, we evaluated contributions of neuroinflammation to the disease development.

Methods and Algorithms: At preclinical (age 20 days), early (5 months), and advanced (18 months) stages of AD-like pathology, in the prefrontal cortex of OXYS and Wistar (control) rats, we evaluated (i) the state of microglial and neuronal cells by histological and immunohistochemical analysis; (ii) differences in the cell-specific (astrocytes, microglia and neurons) gene expression according to RNA sequencing (RNA-Seq) data; (iii) the expression of the main genes of cytokines, chemokines and components of the complement system by RT-PCR.

Results: The number of microglial cells and the expression of the genes involved in immune processes in the cortex of OXYS rats at preclinical stage of the disease indicate the absence of inflammation and increased phagocytic activity, possibly associated with the elimination of "transitional" cells by apoptosis during the formation of interneuronal contacts. By the age of 5 mo the level of mRNA of most of the genes in OXYS rats was increased on the background of neurodegenerative changes. In addition, the changes in expression of microglia-specific genes in OXYS rats were functionally associated with innate immune response and NF- $\mathrm{B}$ pathway. By the age of 18 mo the number of microglial cells was increased and the changes in expression of microglia-specific genes in OXYS rats were associated with innate immune response, response to cytokine and phagocytosis. Notably, 18-mo animals did not differ in the density of microglia and the number of activated microglial cells, which indicates absence of hyperactivation of microglia in OXYS rat cortex at advanced stage of AD-like pathology. However, the number of macrophage cells in OXYS rats was higher than in Wistar rats which may reflect increased need for microglia in conditions of progressive neurodegeneration. This is indicated by a decrease in the expression of the immune system genes and by changes in expression of cell-specific genes associated with regulation of angiogenesis and signal transduction in astrocytes and synaptic transmission in neurons.

Conclusion: These results suggest that dysregulation of the immune system function and the loss of neuroprotective properties of microglia and astrocytes may contribute to the development of AD-like pathology in OXYS rats.

Acknowledgements: This work was supported by grant from the Russian Foundation for Basic Research (project No. 18-015-00320). 